\title{
A model of mitigating risk for IT organisations
}

\begin{abstract}
IT organisations are facing risks that make IT projects not able to meet up planned schedule and quality. Hence, there is the need for risk mitigation in IT organisations to support IT team members to mitigate risk. This paper is a contribution to propose a risk mitigation model to provide expert assistance during IT risk mitigation. By assisting team members and decision makers to identify risks associated with in IT process by providing risk documentation to estimate the probability and impact of IT risks. The risk mitigation model can also introduce risk advice and suggest alternatives to treat the risk by providing support for the reuse of knowledge gained from past IT projects. The risk mitigation model will assist IT organisations in mitigating risk and making right decisions using a risk repository as a medium to provide support for practitioners in the mitigation of mainly operational and technical risk.
\end{abstract}

Keyword: IT project management; IT risk mitigation; Risk; Risk management 